\title{
Experimental Analysis of Dense Multipath Components in an Industrial Environment
}

\author{
Emmeric Tanghe, Davy P. Gaillot, Martine Liénard, Luc Martens, Member, IEEE, and \\ Wout Joseph, Senior Member, IEEE
}

\begin{abstract}
This work presents an analysis of Dense Multipath Components (DMC) in an industrial workshop. Radio channel sounding was performed with a vector network analyzer and virtual antenna arrays. The specular and dense multipath components were estimated with the RiMAX algorithm. The DMC covariance structure of the RiMAX data model was validated. Two DMC parameters were studied: the distribution of radio channel power between specular and dense multipath, and the DMC reverberation time. The DMC power accounted for 23 to $70 \%$ of the total channel power. A significant difference between DMC powers in line-of-sight and non-line-of-sight was observed, which can be largely attributed to the power of the line-of-sight multipath component. In agreement with room electromagnetics theory, the DMC reverberation time was found to be nearly constant. Overall, DMC in the industrial workshop is more important than in office environments: it occupies a fraction of the total channel power that is 4 to $13 \%$ larger. The industrial environment absorbs on average $29 \%$ of the electromagnetic energy compared to $45-51 \%$ for office environments in literature: this results in a larger reverberation time in the former environment. These findings are explained by the highly cluttered and metallic nature of the workshop.
\end{abstract}

Index Terms-channel characterization and modeling, dense multipath components, channel sounding, industrial environment

\section{INTRODUCTION}

$\mathbf{O}$ VER the course of the last decade, the physical view of the radio channel has undergone an important change. Before that, the radio channel was commonly considered to be a collection of only specular multipath components or plane waves, see e.g. [1]. These specular paths have well-defined discrete locations in the different radio channel dimensions (e.g., space, frequency, time, etc.). Nowadays, it is known that part of the radio channel is also continuous across these dimensions. This part is put under the umbrella of dense multipath components [2], [3]. Among other sources, these dense multipath components originate from distributed diffuse

E. Tanghe, L. Martens, and W. Joseph are with Ghent University/iMinds, Department of Information Technology, B-9050 Ghent, Belgium, e-mail: emmeric.tanghe@intec.ugent.be.

D. P. Gaillot and M. Liénard are with University of Lille 1, Institute of Electronics, Microelectronics, and Nanotechnology, F-59655 Villeneuve d'Ascq, France, e-mail: davy.gaillot@univ-lille1.fr.

E. Tanghe is a Post-Doctoral Fellow of the FWO-V (Research Foundation Flanders, Belgium). This work was financially supported by the INTERREG IV project WiSE, the Region Nord Pas-de-Calais and the French Ministry of Research as part of the CISIT (International Campus on Safety and Intermodality in Transportation Systems) project (France), and the FWO-V project G.0325.11N. The authors would also like to acknowledge the scientific input of the COST IC1004 action on Cooperative Radio Communications for Green Smart Environments. scattering on electrically small objects. However, the definition is broader: the philosophy of dense multipath components is to include all radio channel energy - regardless of their exact propagation mechanics - that cannot be associated with the specular multipath components [4], [5]. Hence, this means that dense multipath components may also comprise multipath components that would be categorized as specular multipath from a physical point of view, but cannot be reliably detected because they are too weak and too close to other multipath components in the angular and delay domains. Because of this, only the average power of dense multipath components across these domains can be modeled, while their phase is inaccessible for estimation. This contrasts the specular multipath components where both the power and the phase of the path can be obtained. Therefore, the dense and specular multipath components are often also referred to as the incoherent and coherent parts of the radio channel, respectively. Because the available information and modeling strategies of dense and specular multipath components differ, well-known radio channel parameters have to now be evaluated for both types of multipath separately. The effect of dense multipath components has been investigated on, e.g., the angular channel characteristics in [4], [5], the polarization channel characteristics in [6], [7], the multipath clustering behavior in [8], and the channel capacity in [9].

Dense multipath components have already been investigated in office environments [4], [5], [6], [8]. This work presents - to the best of the authors' knowledge - the first characterization of dense multipath components in an industrial environment, specifically a workshop for shipping container restoration. The parameters under investigation are the fractional power of the dense multipath components and their reverberation time. Their characterization is based on narrowband channel sounding around a $3 \mathrm{GHz}$ center frequency. The specular and dense multipath components are estimated from the channel sounding data by means of the RiMAX framework, i.e., an iterative maximum-likelihood multipath search algorithm [2]. The RiMAX algorithm is particularly suitable for the topic of this paper because it is built on a data model that allows for both specular and dense multipath components. In contrast, estimation algorithms such as Space-Alternating Generalized Expectation-Maximization (SAGE) [10] and Estimation of Signal Parameters via Rotational Invariance Techniques (ESPRIT) [11] historically only assumed specular components in their data model and have not been widely modified yet to account for dense multipath [12]. Although scarce, there exist a number of publications ana- 
lyzing multipath propagation in industrial environments, e.g. [13], [14], [15], [16], [17]. However, the novelty of this paper lies in the fact that it presents the first attempt at separating specular and dense multipath in industrial radio channels.

\section{MeAsurements AND DATA PROCESSING}

\section{A. Measurement environment}

The industrial environment under consideration is a workshop for reparation of intermodal shipping containers. The workshop consists of a single room with dimensions $20.4 \mathrm{~m}$ (length) by $22.0 \mathrm{~m}$ (width) by $4.8 \mathrm{~m}$ (height). The building materials for the walls are a collection of bricks, concrete slabs, steel plates, steel industrial doors, and glass windows. The floor and ceiling are made up of concrete slabs and corrugated steel panels, respectively. The workshop's inventory consists largely of metal container parts, machinery, and small cranes. It is noted that the workshop is intended for manual labor and as such no automated industrial processing lines are present. Fig. 1 shows a photo of the measurement environment, in which the highly cluttered, predominantly metallic nature of the workshop's inventory is clearly apparent.

\section{B. Channel sounding procedure}

Narrowband ${ }^{1}$ frequency-domain channel sounding measurements were performed in the industrial environment under consideration. A Vector Network Analyzer (VNA) of type Rohde \& Schwarz ZNB8 was used to probe the radio channel in a $100 \mathrm{MHz}$ bandwidth centered around $3 \mathrm{GHz}$. In this frequency band, $M_{F}=256$ uniformly spaced frequency points were sampled. The feeder cables for the transmitting and the receiving antenna of type Huber+Suhner S-04212-B were included in the VNA calibration so they do not appear in the measurement data. At both link ends, a virtual antenna array was created by an automated positioning system. A virtual antenna array does not suffer from antenna coupling which perturbs the radio channel measurements. At both transmit $(\mathrm{T})$ and receive $(\mathrm{R})$ side, the virtual array was a planar horizontal Uniform Circular Array (UCA) consisting of $M_{T}=M_{R}$ $=8$ antenna elements. The inter-element spacing was 0.45 times the wavelength at the largest measurement frequency of $3.050 \mathrm{GHz}$. For each position of transmit and receive antenna, the VNA took 10 sweeps of the frequency range to be used for measurement noise reduction when applying the RiMAX algorithm. As transmitting and receiving antenna, broadband omnidirectional discone antennas of type ElectroMetrics EM-6116 were used. Both antennas were $1.50 \mathrm{~m}$ above ground level during measurements. Measurements were done outside of regular working hours because frequencyswept measurements with virtual antenna arrays require a static radio channel without movement (in contrast to timedomain measurements which allow for faster acquisition times and which can also capture time-variant channels [18], [19]).

\footnotetext{
${ }^{1}$ Narrowband in this context means a small relative bandwidth, i.e., the ratio of the measurement bandwidth to the center frequency. This ensures that the electrical dimensions of the antenna arrays do not change much over the measurement bandwidth. This property is used by the multipath estimation algorithm to separate the spatial and frequency dimensions in order to keep the algorithm computationally viable.
}

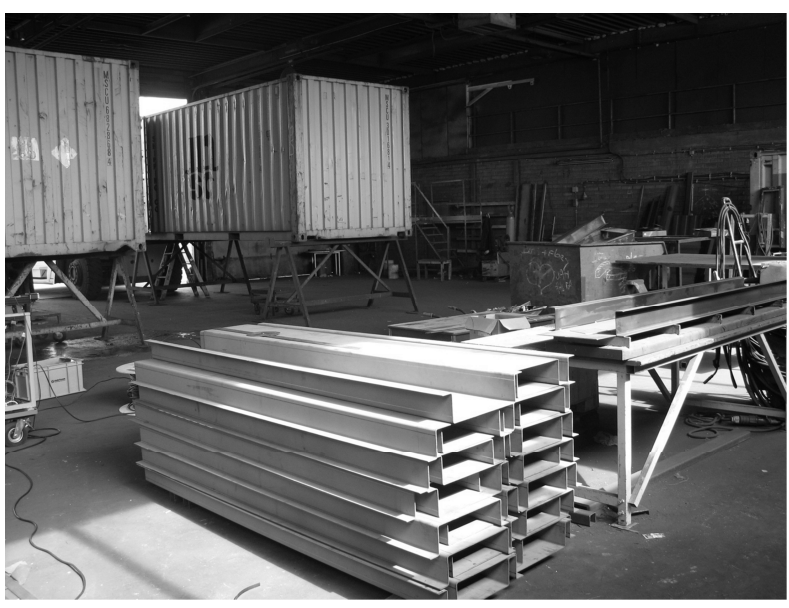

Figure 1. Measurement environment

Fig. 2 presents a floor plan of the measurement environment with transmitting $(\mathrm{Tx})$ and receiving $(\mathrm{Rx})$ antenna locations. In total, $12 \mathrm{Tx}-\mathrm{Rx}$ links were measured: the $\mathrm{Tx}$ remained at a fixed location while the $\mathrm{Rx}$ was moved to different locations between measurements $\left(\mathrm{Rx}_{i}\right.$ for $i=1, \ldots, 12$ in Fig. 2). Measurement were done for three different Tx-Rx link shadowing conditions: Line-of-Sight (LoS, circles in Fig. 2), Obstructed Line-of-Sight (OLoS, squares), and Non-Line-ofSight (NLoS, triangles). The distinction between OLoS and NLoS was made as follows: in the former case the LoS component was blocked by an obstacle such as a small crane, which contains gaps through which the Tx and $\mathrm{Rx}$ can still partially see each other. In the latter case the LoS component was visually fully blocked by an obstacle such as a container.

\section{Estimation of specular and dense multipath components}

1) RiMAX data model: The measured array response vector $\boldsymbol{h} \in \mathbb{C}^{M_{R} M_{T} M_{F} \times 1}$ can be written as the sum of a deterministic

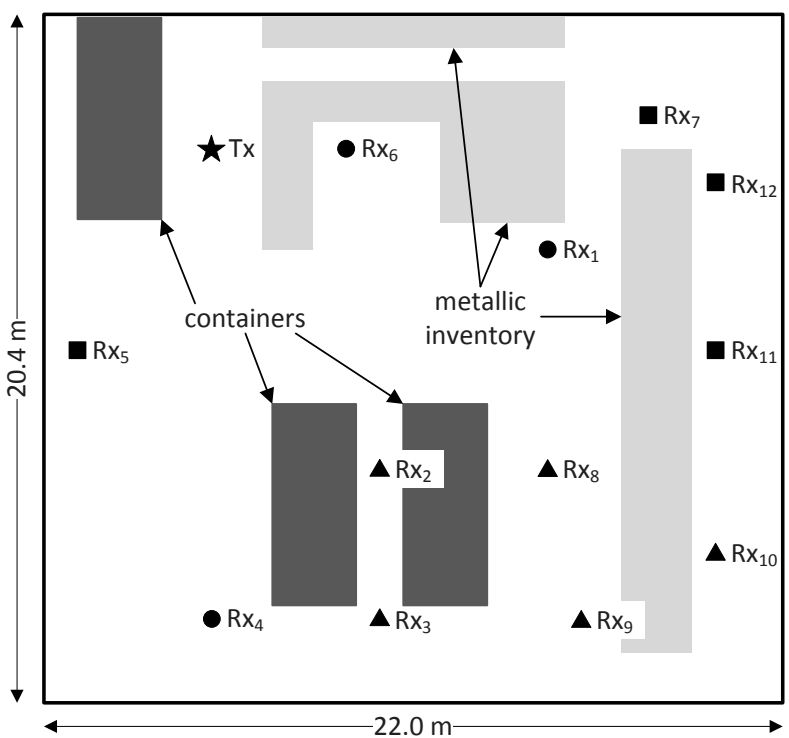

Figure 2. Floor plan with Tx and Rx locations (circle: LoS, square: OLoS, triangle: NLoS) 
part $\boldsymbol{s}\left(\boldsymbol{\theta}_{\boldsymbol{s}}\right)$ and a stochastic part $\boldsymbol{d}\left(\boldsymbol{\theta}_{\boldsymbol{d}}\right)$, i.e., $\boldsymbol{h}=\boldsymbol{s}\left(\boldsymbol{\theta}_{\boldsymbol{s}}\right)+\boldsymbol{d}\left(\boldsymbol{\theta}_{\boldsymbol{d}}\right)$. The deterministic part is a function of the channel's Specular Multipath Components (SMC). The SMC parameters are grouped into the parameter array $\boldsymbol{\theta}_{\boldsymbol{s}}$. The stochastic part is determined by the Dense Multipath Components (DMC) as well as the thermal noise inherent to the measurement equipment. The DMC and noise parameters are grouped into the parameter array $\boldsymbol{\theta}_{\boldsymbol{d}}$. Based on the principle of maximum entropy in Multiple-Input Multiple-Output (MIMO) channel modeling, it is assumed that $\boldsymbol{h}$ is a realization of a multivariate complex Gaussian process with probability density function [20]:

$$
\begin{aligned}
p\left(\boldsymbol{x} ; \boldsymbol{\theta}_{\boldsymbol{s}}, \boldsymbol{\theta}_{\boldsymbol{d}}\right)=\pi^{-M} \operatorname{det}\left(\boldsymbol{R}\left(\boldsymbol{\theta}_{\boldsymbol{d}}\right)\right)^{-1} \\
\quad \cdot \exp \left[-\left(\boldsymbol{x}-\boldsymbol{s}\left(\boldsymbol{\theta}_{\boldsymbol{s}}\right)\right)^{H} \boldsymbol{R}^{-1}\left(\boldsymbol{\theta}_{\boldsymbol{d}}\right)\left(\boldsymbol{x}-\boldsymbol{s}\left(\boldsymbol{\theta}_{\boldsymbol{s}}\right)\right)\right]
\end{aligned}
$$

where $M=M_{R} M_{T} M_{F}$. The covariance matrix $\boldsymbol{R}\left(\boldsymbol{\theta}_{\boldsymbol{d}}\right)$ depends only on the DMC + noise parameters $\boldsymbol{\theta}_{\boldsymbol{d}}$. The data model of the RiMAX estimation framework assumes the DMC is Kronecker-separable in the spatial and frequency domains. Mathematically, the covariance matrix $\boldsymbol{R}\left(\boldsymbol{\theta}_{\boldsymbol{d}}\right)$ has the following structure [2]:

$$
\boldsymbol{R}\left(\boldsymbol{\theta}_{\boldsymbol{d}}\right)=\boldsymbol{I}_{\boldsymbol{M}_{\boldsymbol{R}}} \otimes \boldsymbol{I}_{\boldsymbol{M}_{\boldsymbol{T}}} \otimes \boldsymbol{R}_{\boldsymbol{f}}+\alpha_{0} \boldsymbol{I}_{\boldsymbol{M}}
$$

where $\boldsymbol{I}_{k}$ is the identity matrix of size $k$. According to (2), RiMAX's data model assumes DMC that is spatially white at transmit and receive side (i.e., has constant angular power densities). Additionally, measurements are corrupted by complex additive white Gaussian noise with power $\alpha_{0}$. Furthermore, the DMC is assumed correlated and wide-sense stationary in the frequency domain, which implies that the frequency covariance matrix $\boldsymbol{R}_{f}$ has a Toeplitz structure. The DMC power $\psi(\tau)$ is modeled as an exponential decay as function of delay $\tau$ [2]. An exponential decay is commonly found in highly scattering environments such as for example reverberation chambers [21]:

$$
\psi(\tau)=\alpha_{1} \exp \left[-\frac{\tau-\tau_{d}}{\tau_{r}}\right] \quad\left(\tau>\tau_{d}\right)
$$

From (3), the frequency covariance matrix is calculated as $\boldsymbol{R}_{\boldsymbol{f}}=\operatorname{toep}\left(\boldsymbol{\kappa}, \boldsymbol{\kappa}^{H}\right)$, where $\boldsymbol{\kappa} \in \mathbb{C}^{M_{F} \times 1}$ is a sampled version of the power spectral density associated with $\psi(\tau)$ (i.e., the Fourier transform of (3)). Based on the capabilities of the measurement system in Section II-B and equations (2) and (3), the following structure for SMC and DMC + noise parameter arrays is set:

$$
\begin{aligned}
& \boldsymbol{\theta}_{\boldsymbol{s}}=\left[\begin{array}{c}
\varphi_{A} \\
\vartheta_{A} \\
\varphi_{D} \\
\vartheta_{D} \\
\tau_{A} \\
\gamma
\end{array}\right] \begin{array}{l}
\leftarrow \text { SMC azimuth of arrival } \\
\leftarrow \text { SMC elevation of arrival } \\
\leftarrow \text { SMC azimuth of departure } \\
\leftarrow \text { SMC time of arrival } \\
\leftarrow \text { SMC complex amplitude }
\end{array} \\
& \boldsymbol{\theta}_{\boldsymbol{d}}=\left[\begin{array}{c}
\alpha_{1} \\
\tau_{d} \\
\tau_{r} \\
\alpha_{0}
\end{array}\right] \begin{array}{l}
\leftarrow \text { DMC peak power } \\
\leftarrow \text { DMC onset time } \\
\leftarrow \text { DMC reverberation time } \\
\leftarrow \text { noise power }
\end{array}
\end{aligned}
$$

In (4), $\theta_{s}$ is a $6 \times N$ matrix where $N$ is the number of specular multipath components. Each row of $\boldsymbol{\theta}_{\boldsymbol{s}}$ contains the corresponding specular parameter for each of the $N$ specular paths. Furthermore, $\boldsymbol{\theta}_{\boldsymbol{d}}$ is a $4 \times 1$ vector containing the DMC and noise parameters. Finally, we note that the RiMAX framework is an iterative maximum-likelihood algorithm: the estimates $\hat{\boldsymbol{\theta}}_{\boldsymbol{s}}$ and $\hat{\boldsymbol{\theta}}_{\boldsymbol{d}}$ of the SMC and DMC + noise parameter arrays are determined such that they maximize the likelihood of observing the measured response $\boldsymbol{h}$.

2) Model order selection and reliability of specular paths: A major topic of this paper is the power distribution between SMC and DMC. Because of this, the issue of model order selection or the determination of the number of specular paths $N$ should be treated with care. The usual approach to model order selection is to use algorithms such as the Akaike information criterion or the minimum description length which calculate the log-likelihood of candidate models with different $N$ to decide on its value [22]. Instead in this paper, we use the approach outlined in [2] that is based on the estimated power of specular paths. An important feature of the RiMAX algorithm is that it provides as a by-product an estimate of the Fisher Information Matrix (FIM). For asymptotically uncoupled parameters, the diagonal elements of the inverse of the FIM are variance estimates of the channel parameters in (4). It is possible to associate a Signal-to-Noise Ratio (SNR) with the estimated specular path magnitudes: a specular path $p$ with estimated complex amplitude $\hat{\gamma}_{p}$ has an SNR $\hat{\rho}_{p}$ equal to

$$
\hat{\rho}_{p}=\frac{\left|\hat{\gamma}_{p}\right|^{2}}{\operatorname{var}\left(\left|\hat{\gamma}_{p}\right|\right)}
$$

In (5), $\operatorname{var}(\cdot)$ is the variance of its argument. The specular path $p$ is deemed unreliable and is removed from further analysis if its SNR $\hat{\rho}_{p}$ is too small. It can be derived that the $\left|\hat{\gamma}_{p}\right|$ estimator follows a half-normal distribution (i.e., the distribution of the absolute value of a normally distributed random variable with zero mean) with variance $\operatorname{var}\left(\left|\hat{\gamma}_{p}\right|\right)$ [2]. The SNR $\hat{\rho}_{p}$ in (5) then follows a chi-squared distribution with one degree of freedom $\left(\chi_{1}^{2}\right)$. We retain path $p$ if its estimated SNR is larger than the 90th percentile of $\chi_{1}^{2}$, equal to 2.71 or $4.32 \mathrm{~dB}$. The RiMAX algorithm is iterative: in each iteration, a fixed number of new specular paths are estimated from the measured channel response. The number of new paths per iteration can be chosen arbitrarily and was set to five. If all five paths in one iteration fail the SNR threshold of $4.32 \mathrm{~dB}$, then we consider the channel to be exhausted of reliable specular paths and the RiMAX algorithm is stopped. We further note that model order selection based on (5) is more suited for this paper's topic than selection based on information criteria. The latter calculate an optimal value for the size of the signal subspace as a whole without deciding on the reliability of individual specular paths. However, the path SNR method checks each individual path for its reliability. This is more in line with the philosophy of dense multipath: dense multipath can also comprise specular paths that cannot be resolved reliably due to the channel sounder's limited aperture(s).

Fig. 3 visualizes the model order selection for the $T x-\mathrm{Rx}_{1}$ 


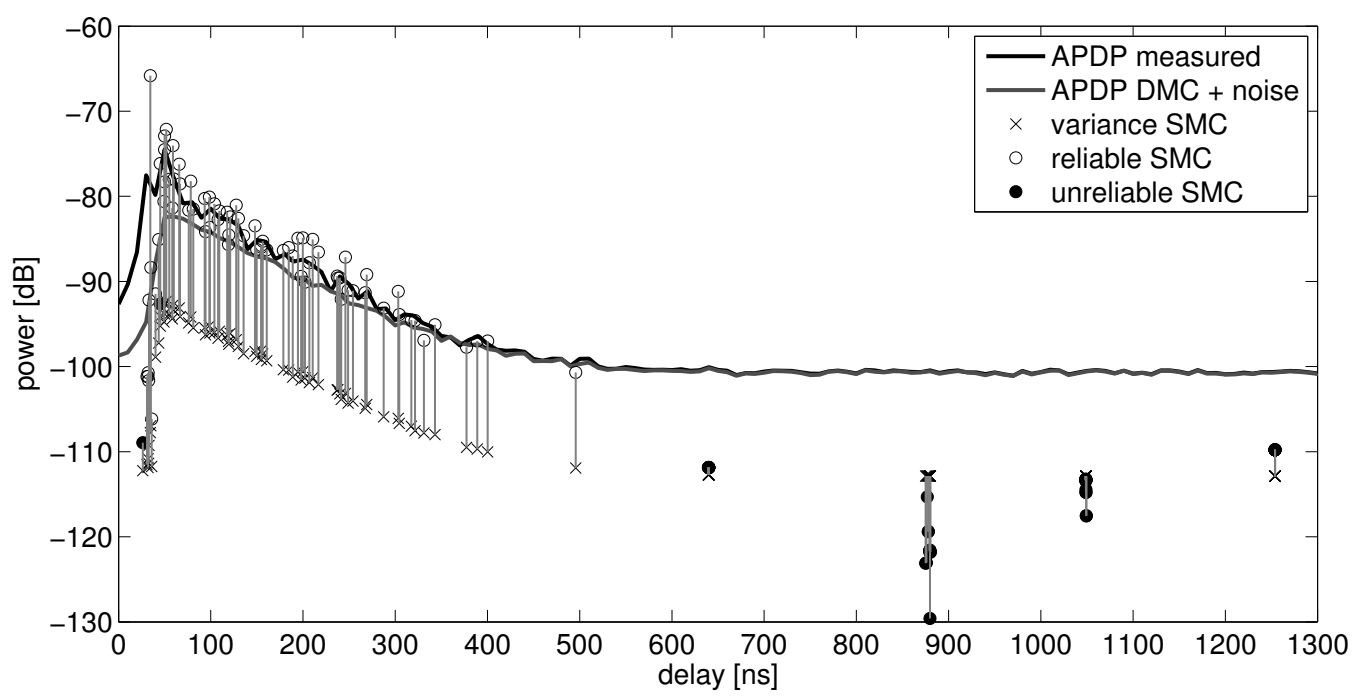

Figure 3. SMC and DMC + noise in the delay domain for the $T x-\mathrm{Rx}_{1}$ link

link. Shown are the measured Averaged Power Delay Profile (APDP), the estimated SMC, and the APDP of the DMC + noise part after subtracting the (reliable) SMC from the channel response. In Fig. 3, the estimated SMC are shown as circles. Vertical lines connect each specular path with the variance of the path's magnitude, shown as crosses. The vertical lines directly represent the SNR $\hat{\rho}_{p}$ in (5) in $\mathrm{dB}$. If the SNR is less than $4.32 \mathrm{~dB}$ then the corresponding path is considered to be unreliable, which is indicated by a filled circle in Fig. 3. Specular paths for which the SNR is greater than $4.32 \mathrm{~dB}$ are reliable and thus retained: these are indicated by an empty circle. We note that the choice of the threshold for the path SNR is argued but still in part arbitrary, as is the case with any criterion. Despite this, our choice appears to work well for our measurement data: for example in Fig 3, SMC that are clearly in the measurement noise part (delays of $500 \mathrm{~ns}$ and larger) are all rejected by the SNR criterion. For the $\mathrm{Tx}-\mathrm{Rx}_{1}$ link in Fig. 3, the RiMAX algorithm stopped with 50 SMC rejected and 75 SMC retained. The model order selection procedure for this link thus resulted in $N=75$ specular paths. Fig. 4 shows the 75 SMC of the $T x-\mathrm{Rx}_{1}$ link, where the length of the plotted rays is proportional to their power in $\mathrm{dB}$. The three most powerful SMC end in diamond markers: they are the LoS component, the ground-reflected ray with azimuth angles close to those of the LoS component, and the ray reflected off the top wall.

3) Validity of the DMC + noise covariance model: This section aims to analyze the goodness-of-fit of the simple DMC + noise covariance structure (2) to our industrial channel sounding data. Following the estimation of the SMC and DMC + noise parameter arrays $\hat{\boldsymbol{\theta}}_{s}$ and $\hat{\boldsymbol{\theta}}_{\boldsymbol{d}}$ in the previous sections, the deterministic and stochastic parts of the measured channel $\boldsymbol{h}$ can be reconstructed as follows:

$$
\begin{aligned}
& \hat{\boldsymbol{s}}=\boldsymbol{A}\left(\hat{\boldsymbol{\varphi}}_{\boldsymbol{A}}, \hat{\vartheta}_{\boldsymbol{A}}, \hat{\boldsymbol{\varphi}}_{\boldsymbol{D}}, \hat{\vartheta}_{\boldsymbol{D}}, \hat{\boldsymbol{\tau}}_{\boldsymbol{A}}\right) \hat{\gamma}^{T} \\
& \hat{d}=\boldsymbol{h}-\hat{\boldsymbol{s}}
\end{aligned}
$$

In (6), $\boldsymbol{A} \in \mathbb{C}^{M_{R} M_{T} M_{F} \times N}$ is an appropriate steering matrix

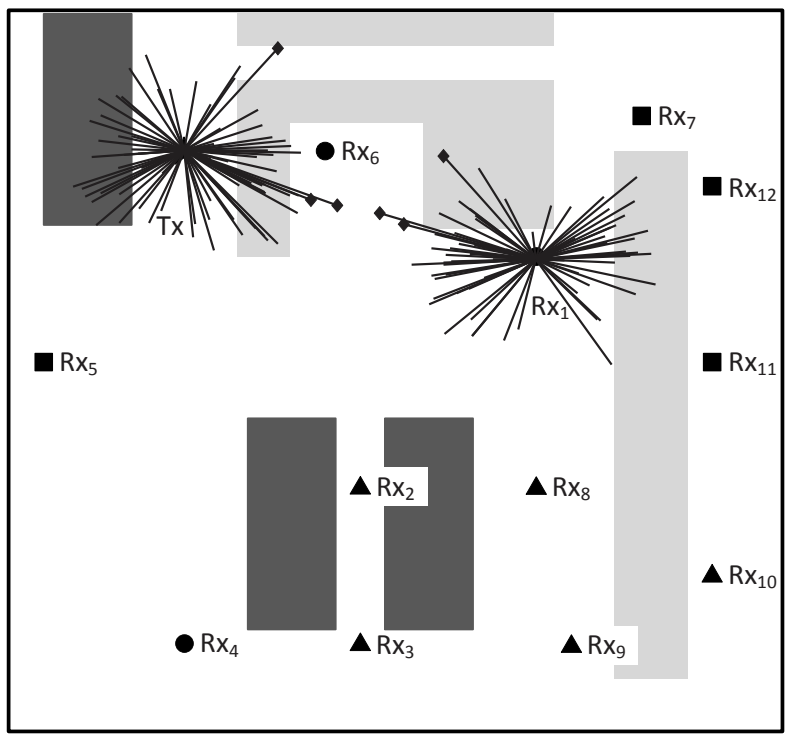

Figure 4. SMC of the Tx-Rx 1 link (the three most powerful SMC end in a diamond marker)

accounting for the array geometries and frequency settings of the channel sounder. The goodness-of-fit analysis is started by performing a decorrelation transformation on the stochastic part $\hat{d}$ using the reconstructed covariance matrix $\boldsymbol{R}\left(\hat{\boldsymbol{\theta}}_{\boldsymbol{d}}\right)$ in (2):

$$
\hat{d}_{w}=R^{-1 / 2}\left(\hat{\theta}_{d}\right) \hat{d}
$$

If the covariance structure (2) is a perfect fit for the stochastic part then the decorrelation transformation (8) is a whitening transformation. In this case $\hat{\boldsymbol{R}}_{\boldsymbol{w}}=\mathbb{E}\left\{\hat{\boldsymbol{d}}_{\boldsymbol{w}} \hat{\boldsymbol{d}}_{\boldsymbol{w}}{ }^{H}\right\}$, i.e., the covariance matrix associated with $\hat{d}_{\boldsymbol{w}}$, is the identity matrix. Due to the usual high dimensionality of channel sounding data, it is more than often impossible to obtain a well-conditioned estimate of $\hat{\boldsymbol{R}}_{\boldsymbol{w}}$. For example, the size of $\hat{\boldsymbol{R}}_{\boldsymbol{w}}$ is $M_{R} M_{T} M_{F} \times M_{R} M_{T} M_{F}=16384 \times 16384$ for our channel sounding data. In contrast only ten channel observations (ten VNA frequency sweeps in Section II-B) are available to 
estimate the expectation $\mathbb{E}\{\cdot\}$, which is largely insufficient. To alleviate this issue, it is further assumed that the spatial and frequency dimensions of $\hat{\boldsymbol{R}}_{\boldsymbol{w}}$ are separable: this is justified given the narrowband character of the channel sounding procedure. This is mathematically given by $\hat{\boldsymbol{R}}_{w}=\hat{\boldsymbol{R}}_{w, s} \otimes \hat{\boldsymbol{R}}_{w, f}$, with:

$$
\begin{aligned}
\hat{\boldsymbol{R}}_{\boldsymbol{w}, \boldsymbol{s}} & =\frac{1}{M_{F}} \mathbb{E}\left\{\hat{\boldsymbol{D}}_{\boldsymbol{w}, \boldsymbol{s}} \hat{\boldsymbol{D}}_{\boldsymbol{w}, \boldsymbol{s}}{ }^{H}\right\} \\
\hat{\boldsymbol{R}}_{\boldsymbol{w}, \boldsymbol{f}} & =\frac{1}{M_{R} M_{T}} \mathbb{E}\left\{\hat{\boldsymbol{D}}_{\boldsymbol{w}, \boldsymbol{f}} \hat{\boldsymbol{D}}_{\boldsymbol{w}, \boldsymbol{f}}{ }^{H}\right\}
\end{aligned}
$$

$\hat{\boldsymbol{R}}_{w, s}$ and $\hat{\boldsymbol{R}}_{\boldsymbol{w}, \boldsymbol{f}}$ are the spatial and frequency covariance matrices, respectively. $\hat{\boldsymbol{D}}_{\boldsymbol{w}, \boldsymbol{s}}$ (size $M_{R} M_{T} \times M_{F}$ ) and $\hat{\boldsymbol{D}}_{\boldsymbol{w}, f}$ (size $M_{F} \times M_{R} M_{T}$ ) are reshaped versions of $\hat{d}_{\boldsymbol{w}}$. Each row of $\hat{D}_{w, s}$ (respectively $\hat{D}_{w, f}$ ) contains the channel gain for one Tx-Rx subchannel (respectively one frequency point) across all frequency points (respectively Tx-Rx subchannels). Equations (9) and (10) lead to well-conditioned covariance matrices: $\hat{\boldsymbol{R}}_{\boldsymbol{w}, s}$ and $\hat{\boldsymbol{R}}_{\boldsymbol{w}, f}$ can be interpreted as frequency and spatially averaged versions respectively of the full covariance matrix $\hat{\boldsymbol{R}}_{w}$. Fig. 5 shows the magnitudes in $\mathrm{dB}$ of the elements of $\hat{\boldsymbol{R}}_{w, s}$ and $\hat{\boldsymbol{R}}_{w, f}$ for the Tx-Rx 1 link. It is observed that both the spatial and frequency covariance matrices approach the identity matrix, thereby confirming the validity of the DMC + noise covariance structure (2) for this link.

The proximity of $\hat{\boldsymbol{R}}_{w, s}$ and $\hat{\boldsymbol{R}}_{\boldsymbol{w}, \boldsymbol{f}}$ to the identity matrix is further investigated quantitatively using the Correlation Matrix Distance (CMD) measure [23]. The CMD $d(\boldsymbol{A}, \boldsymbol{B})$ between two covariance matrices $\boldsymbol{A}$ and $\boldsymbol{B}$ of equal size is defined as:

$$
d(\boldsymbol{A}, \boldsymbol{B})=1-\frac{\operatorname{tr}(\boldsymbol{A} \boldsymbol{B})}{\|\boldsymbol{A}\|_{2}\|\boldsymbol{B}\|_{2}} \in[0,1]
$$

In (11) $\operatorname{tr}(\cdot)$ denotes the trace and $\|\cdot\|_{2}$ is the Frobenius norm. $d(\boldsymbol{A}, \boldsymbol{B})$ is zero for equal covariance matrices and becomes one if the matrices differ maximally. For all $12 \mathrm{Tx}-\mathrm{Rx}$ links of our measurement campaign, $d\left(\hat{\boldsymbol{R}}_{\boldsymbol{w}, s}, \boldsymbol{I}_{\left.\boldsymbol{M}_{R} \boldsymbol{M}_{T}\right)}\right)$ varies between 0.03 and 0.05 , while $d\left(\hat{\boldsymbol{R}}_{\boldsymbol{w}, \boldsymbol{f}}, \boldsymbol{I}_{\boldsymbol{M}_{\boldsymbol{F}}}\right)$ ranges between 0.19 and 0.23 . These low values for both CMDs support the whiteness of $\hat{d}_{w}$ under the narrowband assumption and thus by extension the validity of the DMC + noise covariance structure (2) for our measurement data. Finally, the observation

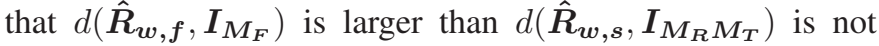
necessarily because the frequency covariance model is a worse fit to our measurement data than the spatial covariance model. We note that the $\hat{\boldsymbol{R}}_{\boldsymbol{w}, \boldsymbol{f}}$ estimator in (10) is less accurate (has larger variance) than the $\hat{\boldsymbol{R}}_{\boldsymbol{w}, s}$ estimator in (9), because $M_{R} M_{T}<M_{F}$. The smaller estimation accuracy of $\hat{\boldsymbol{R}}_{\boldsymbol{w}, \boldsymbol{f}}$ may make this covariance matrix appear to be further away from the identity matrix than $\hat{\boldsymbol{R}}_{w, s}$.

4) Calculation of SMC and DMC power: For the purpose of this section, the reconstructed deterministic part $\hat{s}$ in (6) and stochastic part $\hat{d}$ in (7) are first reshaped as matrices $\hat{\boldsymbol{S}}_{f}$ and $\hat{\boldsymbol{D}}_{f} \in \mathbb{C}^{M_{F} \times M_{R} M_{T}}$. Each column of $\hat{\boldsymbol{S}}_{f}$ (respectively $\hat{D}_{f}$ ) contains the frequency response of the SMC (respectively $\mathrm{DMC}+$ noise) for one Tx-Rx subchannel. The APDPs of the $\mathrm{SMC}$ and $\mathrm{DMC}+$ noise can then be calculated:

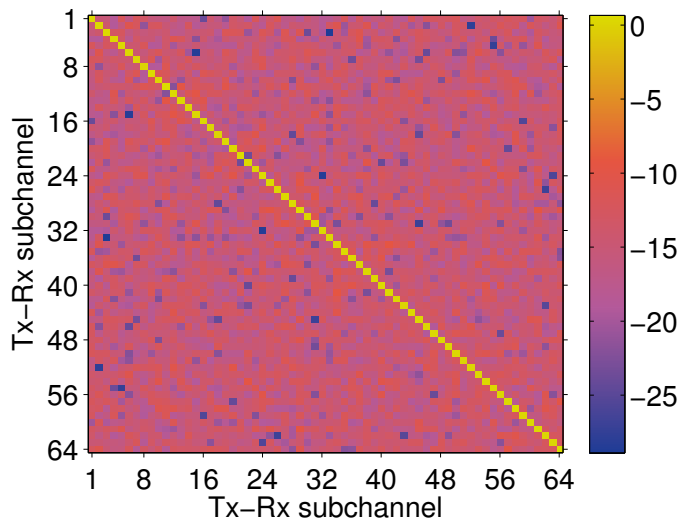

(a) magnitude of $\hat{\boldsymbol{R}}_{w, s}$ in $\mathrm{dB}$

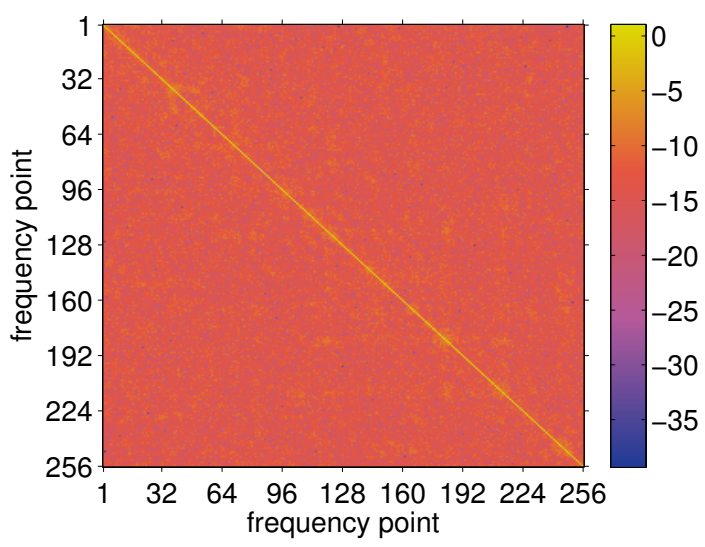

(b) magnitude of $\hat{\boldsymbol{R}}_{\boldsymbol{w}, \boldsymbol{f}}$ in $\mathrm{dB}$

Figure 5. Spatial and frequency covariance matrices of the DMC + noise part of the $\mathrm{Tx}_{\mathrm{R}} \mathrm{Rx}_{1}$ link

$$
\widehat{\operatorname{APDP}}(\mathrm{SMC})=\frac{1}{M_{R} M_{T}} \operatorname{diag}\left(\boldsymbol{F}^{H} \hat{\boldsymbol{S}}_{\boldsymbol{f}}\left(\boldsymbol{F}^{H} \hat{\boldsymbol{S}}_{\boldsymbol{f}}\right)^{H}\right)
$$

$$
\begin{aligned}
& \widehat{\mathrm{APDP}}(\mathrm{DMC}+\text { noise }) \\
& \quad=\frac{1}{M_{R} M_{T}} \operatorname{diag}\left(\mathbb{E}\left\{\boldsymbol{F}^{H} \hat{\boldsymbol{D}}_{\boldsymbol{f}}\left(\boldsymbol{F}^{H} \hat{\boldsymbol{D}}_{\boldsymbol{f}}\right)^{H}\right\}\right)
\end{aligned}
$$

In (12) and (13), $\boldsymbol{F} \in \mathbb{C}^{M_{F} \times M_{F}}$ is the discrete Fourier transform matrix and $\operatorname{diag}(\cdot)$ returns the main diagonal of its matrix argument. The average powers of the SMC and of the DMC + noise for a Single-Input Single-Output (SISO) link can be obtained by summing the APDPs (12) and (13) over all delay bins:

$$
\hat{P}(\mathrm{SMC})=\frac{1}{M_{R} M_{T}} \operatorname{tr}\left(\boldsymbol{F}^{H} \hat{\boldsymbol{S}}_{\boldsymbol{f}}\left(\boldsymbol{F}^{H} \hat{\boldsymbol{S}}_{\boldsymbol{f}}\right)^{H}\right)
$$

$\hat{P}(\mathrm{DMC}+$ noise $)$

$$
=\frac{1}{M_{R} M_{T}} \operatorname{tr}\left(\mathbb{E}\left\{\boldsymbol{F}^{H} \hat{\boldsymbol{D}}_{\boldsymbol{f}}\left(\boldsymbol{F}^{H} \hat{\boldsymbol{D}}_{\boldsymbol{f}}\right)^{H}\right\}\right)
$$


Because only radio channel characteristics are of interest, it is important to modify (15) to remove the contribution of the non-channel effect, i.e., the measurement noise. This is easily done by observing the independence of the DMC and noise processes, and by making use of the RiMAX estimator $\hat{\alpha}_{0}$ for the measurement noise power. The average power of the DMC is then calculated as:

$$
\hat{P}(\text { DMC })=\hat{P}(\text { DMC }+ \text { noise })-M_{F} \hat{\alpha}_{0}
$$

A quantity of interest is the fractional DMC power $\hat{f}_{\mathrm{DMC}}$, i.e., the percentage the DMC contributes to the total channel power:

$$
\hat{f}_{\mathrm{DMC}}=\frac{\hat{P}(\mathrm{DMC})}{\hat{P}(\mathrm{SMC})+\hat{P}(\mathrm{DMC})} \times 100 \%
$$

\section{RESULTS}

Table I lists for each Tx-Rx link a number of channel parameters that are relevant for the topic of this paper. Shown are the number of SMC $N$ obtained with the procedure in Section II-C2 and two DMC-related parameters: the fractional DMC power $f_{\mathrm{DMC}}$ in (17) and the DMC reverberation time $\tau_{r}$ in $(3)^{2}$. The Tx-Rx links are grouped according to their link shadowing circumstances. The DMC-related parameters are discussed in-depth in the following subsections.

\section{A. Fractional DMC power}

From Table I, there is indication that the fractional DMC power $f_{\mathrm{DMC}}$ becomes larger with increased link shadowing: the sample median of $f_{\mathrm{DMC}}$ equals $26.6 \%, 50.0 \%$, and $63.0 \%$ for LoS, OLoS, and NLoS, respectively. Several comparative statistical tests were carried out on the $f_{\text {DMC }}$ dataset in Table I to substantiate this observation. In the following, all tests are carried out with the link shadowing categories (LoS, OLoS, and NLoS) as groups.

A Kruskal-Wallis analysis of variance confirms our observation that there is a difference in the median value of $f_{\text {DMC }}$ between at least two link shadowing groups ( $\mathrm{p}$-value = 0.04). Mann-Whitney $\mathrm{U}$ tests are subsequently performed to search for significant differences in median $f_{\text {DMC }}$ between link shadowing pairs. No significant difference is found between OLoS and both LoS and NLoS (p-values of 0.11 and 0.29 , respectively). The OLoS category can thus be considered as a gray zone between LoS and NLoS when it comes to DMC power: the distinction of an OLoS category for this particular channel parameter may be omitted in the future if continued studies reach the same conclusion. However, a significant difference in in median $f_{\mathrm{DMC}}$ was found between LoS and NLoS (p-value $=0.04$ ). Further investigation shows that this difference can be largely attributed to the fractional power of the LoS component: the power of the LoS path accounts for $21 \%, 34 \%$, and $31 \%$ of the total channel power of the $\mathrm{Tx}-\mathrm{Rx}_{1}, \mathrm{Tx}^{-\mathrm{Rx}_{4}}$, and $\mathrm{Tx}-\mathrm{Rx}_{6}$ links, respectively (calculated with (6) and (14) by using the SMC estimates of only the

\footnotetext{
${ }^{2}$ For increased readability, the hat symbol is omitted from the estimated parameters in the remainder of the paper.
}

LoS component). As mentioned at the start of this section, the sample median of the fractional DMC power is $63 \%$ for NLoS and $27 \%$ for LoS, a difference of $36 \%$. On the other hand, the sample median of the LoS component's fractional power is $31 \%$. Hence, one can conclude that the decrease in fractional DMC power from NLoS to LoS is largely caused by the introduction of a specular LoS component.

Table II presents a comparison of the fractional DMC power with related work in office environments. It has been established that the fractional DMC power strongly depends on the type of environment [9]. Furthermore, the work of [4] also found a strong link between $f_{\mathrm{DMC}}$ and link shadowing category. Our statistical analysis reaches the same conclusion, except for perceived weak differences between OLoS and LoS, and OLoS and NLoS. From Table II, the $f_{\mathrm{DMC}}$ range of the LoS category of our measurements is shifted $13 \%$ up from [4]. The larger $f_{\text {DMC }}$ in the industrial environment may be explained by an increased susceptibility of this environment to diffuse scattering: Fig. 1 shows a highly cluttered environment comprising small metal parts and tools as well as oblong structural steel. Additionally, our measurements were performed at a lower center frequency than in [4]. This means reduced electrical dimensions of objects and, therefore, reduced occurrences of specular reflection in favor of diffuse scattering.

For our measurements, the largest $f_{\mathrm{DMC}}$ range is found for the OLoS category (Table II). This can be explained by the comparatively larger diversity of shadowing objects for this category. While there are no shadowing objects for LoS and shipping containers are the only type of shadowing object for NLoS, in comparison the types of shadowing objects encountered for OLoS are broader: a container edge for the $\mathrm{Tx}-\mathrm{Rx}_{5}$ link, a workbench for $\mathrm{Tx}-\mathrm{Rx}_{7}$, and a small crane for $\mathrm{Tx}^{-} \mathrm{Rx}_{11}$ and $\mathrm{Tx}-\mathrm{Rx}_{12}$. These results suggest that $f_{\mathrm{DMC}}$ may be best treated as a continuous variable and that there appears to be a gradual power transfer from the SMC to the DMC as link shadowing objects become more dense. Furthermore in Table II, the $f_{\mathrm{DMC}}$ range for OLoS in [4] is somewhat smaller than for our measurements: in line with the explanation above, this may be because there is only one type of OLoS shadowing object in [4], i.e., a flight of stairs.

For the NLoS category, the values of $f_{\mathrm{DMC}}$ in [4] are clearly larger compared to [6] (in the latter a mix of OLoS and NLoS measurements were made). This may have to do with the fact that - contrary to [6] - the transmitter and receiver were on different floors in [4]. The authors of [4] state that this creates a harsh NLoS environment subject to mostly continuous propagation phenomena, for which also part of the SMC cannot be detected due to limited measurement resolution. The NLoS scenario in this paper is more readily comparable to the NLoS scenario in [6]: both have transmitter and receiver on the same floor and while the NLoS situation in [6] is created by brick and plaster walls, the containers (seen in Fig. 1 on the left and in Fig. 2 at the bottom) create a similar NLoS obstruction situation (although not obstructing the entire distance between floor and ceiling like a wall does). The NLoS $f_{\text {DMC }}$ values in this paper are slightly larger than those found in [6], despite the NLoS obstruction not reaching from floor to 


\begin{tabular}{|c|c|c|c|c|c|c|c|c|c|c|c|c|}
\hline \multirow{2}{*}{$\begin{array}{r}\text { link shadowing } \\
\text { Rx number }\end{array}$} & \multicolumn{3}{|c|}{ LoS } & \multicolumn{4}{|c|}{ OLoS } & \multicolumn{5}{|c|}{ NLoS } \\
\hline & 1 & 4 & 6 & 5 & 7 & 11 & 12 & 2 & 3 & 8 & 9 & 10 \\
\hline$N[-]$ & 75 & 99 & 93 & 117 & 65 & 41 & 34 & 46 & 42 & 137 & 66 & 45 \\
\hline$f_{\mathrm{DMC}}[\%]$ & 37.5 & 22.9 & 26.6 & 26.9 & 45.3 & 54.7 & 70.2 & 64.4 & 63.9 & 57.1 & 63.0 & 60.1 \\
\hline$\tau_{r}[\mathrm{~ns}]$ & 77.2 & 79.6 & 70.0 & 84.5 & 74.0 & 70.6 & 64.6 & 73.0 & 79.5 & 82.7 & 83.8 & 74.4 \\
\hline
\end{tabular}

Table I

NuMbER OF SMC, FRACTIONAL DMC POWER, AND DMC REVERBERATION TIME FOR ALL TX-RX LINKS

\begin{tabular}{|c|c|c|c|c|c|}
\hline \multirow{2}{*}{ reference } & environment & \multirow{2}{*}{$\begin{array}{c}\text { center } \\
\text { frequency }\end{array}$} & \multicolumn{3}{|c|}{ link shadowing } \\
\cline { 5 - 6 } & & & OLoS & NLoS \\
\hline \hline 4$]$ & open hall in office building & $5.3 \mathrm{GHz}$ & $10-25 \%$ & $35-65 \%$ & $60-90 \%$ \\
{$[6]$} & office building & $3.6 \mathrm{GHz}$ & $\mathrm{n} / \mathrm{a}$ & \multicolumn{2}{|c|}{$20-60 \%$} \\
this paper & industrial workshop & $3.0 \mathrm{GHz}$ & $23-38 \%$ & $27-70 \%$ & $57-64 \%$ \\
\hline
\end{tabular}

Table II

COMPARISON WITH RELATED WORK FOR $f_{\text {DMC }}$

ceiling in our environment. This redemonstrates the prevalence of diffuse scattering in the industrial environment, just as for the LoS category.

\section{B. DMC reverberation time}

Firstly, Figs. 3 clearly shows that the single exponential decay (3) fits the DMC process very well. This contrast the findings of [8], which uses the OLoS office-environment measurement of [4] and where visually about ten DMC clusters are distinguished. Secondly, Table I reveals that the DMC reverberation time $\tau_{r}$ does not change noticeably between link shadowing categories and is even fairly constant across all Tx-Rx links. This observation is backed by a Kruskal-Wallis analysis of variance which found no difference in median $\tau_{r}$ between link shadowing categories ( $\mathrm{p}$-value $=0.55$ ).

The two observations made about DMC in the previous paragraph - a single exponential decay and a reverberation time that is independent of Rx location - is entirely in line with the theory of Room Electromagnetics (RE) [24], [25], [26]. The RE theory borrows from the field of room acoustics and obtains an exponential decay for the diffuse field under these assumptions: the field's intensity does not depend on direction (rich scattering environment) and its energy density is constant across the whole room (valid for rooms that are small enough). The industrial workshop can be deemed to satisfy both of these assumptions. As with room acoustics, RE is a scalar theory and therefore does not account for more than one polarization at Tx and/or Rx. Furthermore, RE provides a simple relationship between the reverberation time $\tau_{r}$, the room's geometry and a single constant describing the room's electromagnetic absorption qualities:

$$
\tau_{r}=\frac{4 V}{c \eta A}
$$

In (18), $V$ and $A$ are the room's volume and area, respectively, $c$ is the speed of light in vacuum, and $\eta$ is the fraction of energy absorbed by the area $A$. Using the industrial workshop's dimensions in Section II-A, we obtain $V=2154.2 \mathrm{~m}^{3}$ and $A=1304.6 \mathrm{~m}^{2}$. From Table I, the median $\tau_{r}$ across all Tx-Rx links is determined as $75.8 \mathrm{~ns}$. Using (18), we then obtain the median absorption coefficient $\eta$ as 0.29 .

The parameter $\eta$ is particularly interesting to compare the reverberation properties of different rooms: unlike the $\tau_{r}$ parameter, $\eta$ is independent of room geometry and solely depends on the average electromagnetic absorption characteristics of the materials present. These materials in turn directly relate to the type of environment. For example for office environments and vertical polarization at both link ends, [24] found $\eta=0.51$ and [26] obtained $\eta=0.45$. In comparison, $\eta=0.29$ in the industrial workshop for the same polarization conditions reveals that the materials of the latter are 16 to $22 \%$ less absorbent. This is easily explained by the highly reflective nature of the materials commonly found in an industrial environment.

\section{Conclusions}

This work presented a statistical analysis of Dense Multipath Components (DMC) in an industrial workshop for shipping container restoration. The analysis was based on radio channel sounding experiments and the RiMAX maximumlikelihood algorithm was used to estimate specular and dense multipath components from the channel sounding data. The DMC covariance structure of the RiMAX data model was found to be a good fit for the workshop's radio channel. Two parameters related to the DMC were investigated: the fractional DMC power, i.e., the percentage of total channel power that can be attributed to dense multipath, and the DMC reverberation time in the time-delay domain. Among other things, the impact of the link shadowing category, i.e., Line-of-Sight (LoS), Obstructed Line-of-Sight (OLoS), or Non-Line-of-Sight (NLoS), on these two DMC parameters was examined. The following results were obtained for the fractional DMC power:

1) The fractional DMC power varies between $23-38 \%$ (LoS), 27-70\% (OLoS), and 57-64\% (NLoS).

2) The difference between the median fractional DMC powers of the NLoS and the LoS categories $(=36 \%$ 
from sample) is statistically significant. Most of this difference can be attributed to the power of the LoS multipath component: the sample median of the LoS component's fractional power is $31 \%$.

3) The fractional DMC power was found to be somewhat higher in the industrial workshop compared to office environments in literature due to the cluttered and metallic nature of the workshop's inventory. A cautious estimate of the difference is 4 to $13 \%$, while acknowledging the possibility that the fractional DMC power may also correlate with other environment parameters that cannot be easily deduced from literature, e.g., room dimensions, Tx-Rx distance, etc.

These results were obtained for the DMC reverberation time:

1) The DMC process in the time-delay domain is welldescribed by a single exponential decay. Furthermore, the DMC reverberation time is nearly constant and thus independent of Rx location. These observations are entirely in line with the theory of room electromagnetics.

2) Room electromagnetics can be used to calculate the average absorption of electromagnetic energy by the environment. The industrial workshop is found to be 16 to $22 \%$ less absorbent compared to office environments in literature. This is due to the metallic and thus highly reflective nature of the materials in the workshop.

\section{REFERENCES}

[1] H. Krim and M. Viberg, "Two Decades of Array Signal Processing Research: the Parametric Approach," IEEE Signal Processing Magazine, vol. 13, no. 4, pp. 67-94, July 1996.

[2] A. Richter, "Estimation of Radio Channel Parameters: Models and Algorithms," Ph.D. dissertation, Technische Universität Ilmenau, Fakultät für Elektrotechnik und Informationstechnik, Ilmenau, DE, 2005.

[3] C. Oestges, N. Czink, P. De Doncker, V. Degli-Esposti, K. Haneda, W. Joseph, M. Liénard, L. Liu, J. Molina-García-Pardo, M. Narandžić, J. Poutanen, F. Quitin, and E. Tanghe, Pervasive Mobile and Ambient Wireless Communications - COST Action 2100. Springer, 2012, ch. Radio Channel Modeling for 4G Networks, pp. 67-148.

[4] J. Poutanen, J. Salmi, K. Haneda, V.-M. Kolmonen, and P. Vainikainen, "Angular and Shadowing Characteristics of Dense Multipath Components in Indoor Radio Channels," IEEE Transactions on Antennas and Propagation, vol. 59, no. 1, pp. 1-9, January 2011.

[5] F. Mani, F. Quitin, and C. Oestges, "Directional Spreads of Dense Multipath Components in Indoor Environments: Experimental Validation of a Ray-Tracing Approach," IEEE Transactions on Antennas and Propagation, vol. 60, no. 7, pp. 3389-3396, July 2012.

[6] F. Quitin, C. Oestges, F. Horlin, and P. De Doncker, "Diffuse Multipath Component Characterization for Indoor MIMO Channels," in European Conference on Antennas and Propagation, Barcelona, ES, April 2010, pp. 1-5.

[7] M. Landmann, K. Sivasondhivat, J.-I. Takada, I. Ida, and R. Thomä, "Polarization Behavior of Discrete Multipath and Diffuse Scattering in Urban Environments at $4.5 \mathrm{GHz}$,' EURASIP Journal on Wireless Communications and Networking, vol. 1, pp. 1-16, January 2007, doi:10.1155/2007/57980.

[8] J. Salmi, J. Poutanen, K. Haneda, A. Richter, V.-M. Kolmonen, P. Vainikainen, and A. F. Molisch, "Incorporating Diffuse Scattering in Geometry-based Stochastic MIMO Channel Models," in European Conference on Antennas and Propagation, Barcelona, ES, April 2010, pp. 1-5.

[9] A. Richter, J. Salmi, and V. Koivunen, "Distributed Scattering in Radio Channels and its Contribution to MIMO Channel Capacity," in European Conference on Antennas and Propagation, Nice, FR, 2006, pp. 1-7.

[10] B. H. Fleury, M. Tschudin, R. Heddergott, D. Dahlhaus, and K. I. Pedersen, "Channel Parameter Estimation in Mobile Radio Environments Using the SAGE Algorithm," IEEE Journal on Selected Areas in Communications, vol. 17, no. 3, pp. 434-450, March 1999.
[11] M. Haardt, "Efficient One-, Two-, and Multidimensional HighResolution Array Signal Processing," Ph.D. dissertation, Technische Universität München, Shaker Verlag GmbH, Aachen, DE, 1996.

[12] E. Tanghe, D. P. Gaillot, W. Joseph, M. Liénard, P. Degauque, and L. Martens, "Robustness of high-resolution channel parameter estimators in the presence of dense multipath components," IET Electronics Letters, vol. 48, no. 2, pp. 130-132, January 2012.

[13] T. S. Rappaport, S. Y. Seidel, and K. Takamizawa, "Statistical Channel Impulse Response Models for Factory and Open Plan Building Radio Communication System Design," IEEE Transactions on Communications, vol. 39, no. 5, pp. 794-807, May 1991.

[14] P. Yegani and C. D. McGillem, "A Statistical Model for the Factory Radio Channel," IEEE Transactions on Communications, vol. 39, no. 10, pp. 1445-1454, October 1991.

[15] D. Hampicke, A. Richter, A. Schneider, G. Sommerkorn, R. S. Thomä, and U. Trautwein, "Characterization of the Directional Mobile Radio Channel in Industrial Scenarios, Based on Wideband Propagation Measurements," in IEEE Vehicular Technology Conference - Fall, vol. 4, Amsterdam, NL, September 1999, pp. 2258-2262.

[16] J. Karedal, S. Wyne, P. Almers, F. Tufvesson, and A. F. Molisch, "A Measurement-Based Statistical Model for Industrial Ultra-Wideband Channels," IEEE Transactions on Wireless Communications, vol. 6, no. 8, pp. 3028-3037, August 2007.

[17] E. Tanghe, W. Joseph, L. Verloock, L. Martens, H. Capoen, K. Van Herwegen, and W. Vantomme, "The industrial indoor channel: Large-scale and temporal fading at 900, 2400, and $5200 \mathrm{mhz}$, IEEE Transactions on Wireless Communications, vol. 7, no. 7, pp. 2740-2751, July 2008.

[18] A. Dezfooliyan and A. M. Weiner, "Evaluation of Time Domain Propagation Measurements of UWB Systems Using Spread Spectrum Channel Sounding," IEEE Transactions on Antennas and Propagation, vol. 60, no. 10 , pp. 4855-4865, October 2012.

[19] — - "Experimental Test-Bed for Studying Multiple Antenna Beamforming over Ultra Wideband Channels up to $12 \mathrm{GHz}$," IEEE Wireless Communications Letters, vol. 1, no. 5, pp. 520-523, October 2012.

[20] M. Debbah and R. R. Müller, "MIMO Channel Modeling and the Principle of Maximum Entropy," IEEE Transactions on Information Theory, vol. 51, no. 5, pp. 1667-1690, May 2005.

[21] P. Besnier and B. Démoulin, Electromagnetic Reverberation Chambers, 1st ed. Wiley, 2011.

[22] Y. Hua, A. Gershman, and Q. Cheng, Eds., High-Resolution and Robust Signal Processing, 1st ed. CRC Press, October 2003.

[23] M. Herdin and E. Bonek, "A MIMO Correlation Matrix based Metric for Characterizing Non-Stationarity," in IST Mobile and Wireless Communications Summit, Lyon, FR, June 2004, pp. 1-5.

[24] J. B. Andersen, J. O. Nielsen, G. F. Pedersen, G. Bauch, and M. Herdin, "Room Electromagnetics," IEEE Antennas and Propagation Magazine, vol. 49, no. 2, pp. 27-33, April 2007.

[25] A. Bamba, W. Joseph, J. B. Andersen, E. Tanghe, G. Vermeeren, D. Plets, J. O. Nielsen, and L. Martens, "Experimental Assessment of Specific Absorption Rate Using Room Electromagnetics," IEEE Transactions on Electromagnetic Compatibility, vol. 54, no. 4, pp. 747757, August 2012.

[26] A. Bamba, W. Joseph, E. Tanghe, G. Vermeeren, and L. Martens, "Circuit Model for Diffuse Multipath and Electromagnetic Absorption Prediction in Rooms," IEEE Transactions on Antennas and Propagation, vol. 61, no. 6, pp. 3292-3301, June 2013.

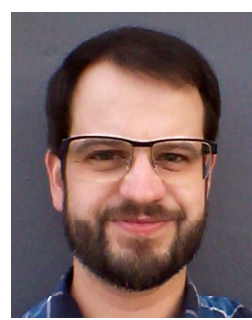

Emmeric Tanghe Emmeric Tanghe was born in Tielt, Belgium on August 31 1982. He received the M. Sc. degree in electrical engineering from Ghent University (Belgium) in July 2005. From September 2005 to May 2011, he was a research assistant at iMinds-UGent/INTEC (Department of Information Technology) of the same university. His scientific work focused on the modeling of indoor and outdoor propagation through field measurements. This work led to a Ph. D. degree in May 2011. Since May 2011, he is post-doctoral researcher at the same institution and continues his work in propagation modeling. Since October 2012, he is a Post-Doctoral Fellow of the FWO-V (Research Foundation - Flanders). 


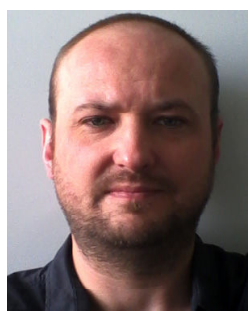

Davy P. Gaillot received a B. S. degree in Mechanical Engineering from Ecole Nationale d'Ingénieurs de Metz (ENIM), Metz, France, in 2002. Simultaneously, he gained a masters degree in Mechanics, Materials, Structures and Processes from the University of Metz, France. After completing his Ph. D. in 2007 at the Department of Materials Science and Engineering at the Georgia Institute of Technology, Atlanta, USA, he was a Post-doctoral fellow at the Institute of Electronics, Microelectronics, and Nanotechnology (IEMN) in Villeneuve d'Ascq, France. Since 2008, he is an Associate Professor at the University of Lille 1 in the IEMN - TELICE group. Prof. Gaillot's research interests include the development of outdoor/indoor radio channel models for localization techniques that are supported by legacy or upcoming millimeter-wave and centimeter-wave wireless network standards. In addition, he focuses on highly diffuse and industrial radio channels for propagation modeling and exposure assessment.

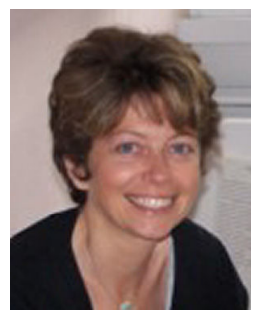

Martine Liénard received the M. Sc. and $\mathrm{Ph}$. D. degrees from Université Lille 1, France, in 1988 and 1993, respectively. Since 1990, she has been with the TELecommunications, Interferences et Compatibilité Electromagnétique (TELICE) Group, Institut dElectronique, de Microélectronique et de Nanotechnologie, where she is currently a professor and the head of TELICE. Her current research interests include both theoretical and experimental prediction of propagation characteristics in confined areas, optimization of the PHY layer including diversity schemes with applications to wireless local area networks, powerline communications, and rail and road transportation communication systems.

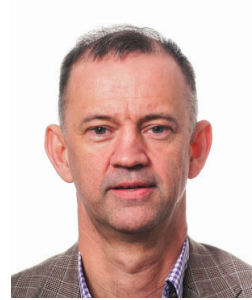

Luc Martens received the M. Sc. degree in electrical engineering from Ghent University (Belgium) in July 1986. From September 1986 to December 1990, he was a research assistant at the Department of Information Technology (INTEC) of the same university. During this period, his scientific work was focused on the physical aspects of hyperthermic cancer therapy. His research work dealt with electromagnetic and thermal modeling, and with the development of measurement systems for that application. This work led to a Ph. D. degree in December 1990. Since 1991, he manages the wireless and cable research group at INTEC. This group is since 2004 part of the iMinds institute. Since April 1993, he is a professor at Ghent University. His experience and current interests are in modeling and measurement of electromagnetic channels, in electromagnetic exposure e.g. around telecommunication networks and systems such as cellular base station antennas, and in energy consumption of wireless networks. He is an author or co-author of more than 300 publications in the domain of electromagnetic channel predictions, dosimetry, exposure systems and health, and wireless communications.

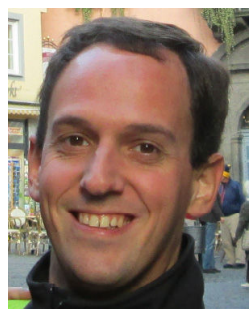

Wout Joseph was born in Ostend, Belgium on October 21 1977. He received the M. Sc. degree in electrical engineering from Ghent University (Belgium) in July 2000. From September 2000 to March 2005, he was a research assistant at the Department of Information Technology (INTEC) of the same university. During this period, his scientific work was focused on electromagnetic exposure assessment. His research work dealt with measuring and modeling electromagnetic fields around base stations for mobile communications, and with health effects of exposure to electromagnetic radiation. This work led to a $\mathrm{Ph}$. D. degree in March 2005. Since April 2005, he is post-doctoral researcher at iMindsUGent/INTEC. From October 2007 to October 2013, he was a Post-Doctoral Fellow of the FWO-V (Research Foundation Flanders). Since October 2009, he is a professor in the domain of experimental characterization of wireless communication systems. His professional interests are electromagnetic exposure assessment, propagation for wireless communication systems, and antennas and calibration. Furthermore, he specializes in wireless performance analysis and quality of experience. 